\title{
Apprendre la nature à l'école primaire française (1867-1909)
}

Pierre Dasi

\section{(2) OpenEdition}

12 Journals

Édition électronique

URL : https://journals.openedition.org/ere/8479

DOI : 10.4000/ere.8479

ISSN : 2561-2271

Éditeur

Centr'ERE

Référence électronique

Pierre Dasi, «Apprendre la nature à l'école primaire française (1867-1909) », Éducation relative à l'environnement [En ligne], Volume 16-2 | 2021, mis en ligne le 14 octobre 2021, consulté le 16 février 2022. URL : http://journals.openedition.org/ere/8479; DOI : https://doi.org/10.4000/ere.8479

Ce document a été généré automatiquement le 16 février 2022.

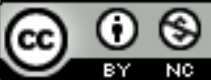

La revue Éducation relative à l'environnement est mise à disposition selon les termes de la Licence Creative Commons Attribution - Pas d'Utilisation Commerciale 4.0 International. 


\title{
Apprendre la nature à l'école primaire française (1867-1909)
}

\author{
Pierre Dasi
}

1 Voilà un mariage qui sonne comme une évidence. Est-il possible même d'imaginer que la mise en ménage de l'éducation et de la nature ne soit pas aussi vieille que l'école ellemême ! Bien avant la proclamation de la Troisième République (Septembre 1870 - Juillet 1940), Aristote avait initié Alexandre le Grand à l'histoire naturelle, Rabelais élevé la nature au rang d'une étude pantagruélique, le pédagogue tchèque Amos Comenius interprété Bacon et préconisé l'observation concrète de la nature. Et puis, Rollin, Diderot, Pestalozzi, Frœbel, sans oublier Rousseau puis Spencer au XIX ${ }^{\mathrm{e}}$ siècle, chacun à leur tour, chez ces pédagogues, la même intuition, la même recommandation: enseigner à l'enfant les faits de nature. Voix d'oracles, voix fécondes. Même sommaire, ce survol tend à montrer que les liens entre l'éducation et la nature s'enfoncent dans les racines de l'histoire pédagogique. Alors quoi? Ces «noces» tardives constitueraient-elles autre chose qu'une simple régularisation de pratiques inhérentes à l'institution scolaire? Difficile d'en douter tant, dans les sources produites par les acteurs des écoles primaires à partir des années 1870, abondent les exercices, les textes littéraires ou scientifiques composés autour du thème de la nature ${ }^{1}$.

\section{Un nouveau champ de recherches}

2 Cette inflexion didactique, l'historien de la culture Maurice Crubellier, l'avait déjà décelée voilà plus de quarante ans (Crubellier, 1978) affirmant même que l'enseignement de la nature constitua un levier majeur du processus d'unification et de laïcisation des esprits. Plus récemment, les travaux de Pierre Kahn sur les sciences (Kahn, 2014) ou sur la leçon de choses (Kahn, 2002) comme ceux de Jean-Pierre Chevalier sur la géographie du primaire (Chevalier, 2003) ont permis de réinterroger cette question de la nature dans le jeu des disciplines scolaires. De leur côté, JeanFrançois Chanet (1996) et Anne-Marie Thiesse (1997) ont montré que, dans les écoles républicaines, la construction du sentiment national français s'était largement appuyée 
sur l'exaltation des paysages naturels. Pour se tenir au plus près des réalités de la classe, ces historiens ne se sont pas contentés de compulser les textes officiels et réglementaires, ils se sont penchés sur les dictées, les rédactions, les cartes, les livres de lecture, les exercices pratiques auxquels étaient confrontés les enfants du primaire, sans jamais négliger les réflexions des fondateurs de l'École républicaine.

3 C'est de cette démarche dont nous nous sommes inspirés pour conduire cette réflexion sur l'avènement de la nature dans les écoles de la Troisième République. Et, en nous adossant à des sources éclectiques mêlant des travaux d'élèves et des extraits de revues pédagogiques comme le Manuel général, en recourant aux manuels scolaires des différentes disciplines publiés entre 1870 et 1914, en examinant le monumental Dictionnaire de Pédagogie, en revenant constamment vers l'incontournable Tour de France par deux enfants, en convoquant les républicains fondateurs de l'école primaire, nous avons été frappés par l'importance des traces imprimées par la nature dans toute cette documentation.

Cette irruption est d'autant plus spectaculaire qu'elle tranche avec le contenu des sources antérieures aux années 1870. Pour s'en convaincre, il suffit de jeter un œil au Manuel général de l'Instruction primaire avant et après cette date. Pas besoin d'y regarder de près pour voir monter la marée discursive charriant un flot de savoirs sur l'environnement des écoliers. À tel point qu'il nous semble possible d'affirmer - dans le prolongement de Maurice Crubellier - que l'importation des représentations de la nature au sein de l'école Ferryste a promu une culture scolaire fortement imprégnée de naturalisme. Qu'il s'agisse de la géographie, des sciences, des mathématiques ou que l'on regarde vers le français ou les arts, toutes ces disciplines se sont emparées de la diversité du vivant pour enrichir leurs contenus. Et toutes ont pu puiser leur inspiration dans le Dictionnaire de pédagogie dont le bataillon de rédacteurs passe au crible tout ce qui relève du champ éducatif. D'une collecte méthodique en direction des sciences de la nature, on rapporte, en vrac, des savants patentés de titres, des vertébrés et des invertébrés, des classifications rigoureuses et une avalanche de lois naturelles. Quoi d'autres? Des injonctions répétées pour améliorer les méthodes culturales (engrais, amendements, irrigation, assainissement, protection des bois et forêts...), des considérations cosmologiques et de multiples savoirs sur des espèces aux noms impossibles : « Helminthes », « Spongiaires » et autres « Rayonnés ». Bien sûr, l'absence dans le Dictionnaire d'une notice exclusivement consacrée au terme de "nature » interroge. Doit-on, pour autant, en conclure qu'il s'agit d'un impensé de la culture scolaire?

5 Ni silence, ni effacement de l'univers, l'absence d'entrée au mot « Nature » laisse le champ libre à une accumulation d'articles constituant une grammaire visant à comprendre les environnements naturels. Empruntant à des champs épistémologiques variés - sciences, philosophie, psychologie, pédagogie, géographie, lettres, arts - les déclinaisons des façons de dire les détails du monde font coexister de multiples représentations de la nature dans les sources du primaire. Là coexistent des idées de nature, des pratiques sociales et des conceptions plurielles qui sont à la fois le reflet et le support du monde environnant. Creuset des perceptions, des routines, des évidences, du quotidien, des choses de la vie, des situations institutionnelles, des figures obligées, tout ce qui concerne la nature se rencontre dans le corpus scolaire. Peut-on alors imaginer que ce foisonnement de réflexions sur les composantes de la nature n'exprime pas l'attachement des pédagogues rénovateurs à cette thématique? Que Ferdinand 
Buisson, maître d'œuvre du Dictionnaire de pédagogie et James Guillaume, son secrétaire de rédaction, n'aient pas jugé nécessaire d'y consacrer un article spécifique - peut-être au risque de réduire l'ampleur et la variabilité du concept de nature - n'empêche pas ces intellectuels-pédagogues de plaider pour une éducation ouverte sur l'extérieur alliant le beau et l'utile dans une juste mise en valeur de la nature. Surtout, cela n'invalide pas la thèse selon laquelle la saisine de la nature a constitué un axe majeur du projet éducatif de l'école de la Troisième République. Jamais formulée avec éclat cette ambition peut apparaître comme un implicite de la pensée des réformateurs de l'enseignement primaire de la Troisième République -, il n'en reste pas moins qu'elle explique, pour une large part, aussi bien la promotion de nouveaux savoirs que la rénovation des pratiques pédagogiques qui sont alors à l'œuvre dans les écoles primaires. En regardant vers l'ensemble des champs cognitifs - de la morale aux mathématiques - nous cherchons à montrer dans cet article que l'entrée de la nature dans les écoles primaires a non seulement renforcé la coloration patriotique du curriculum, mais aussi posé les bases d'une nouvelle éducation dont l'une des finalités a consisté à fabriquer de nouvelles façons d'envisager les relations entre les enfants et la nature. ${ }^{2}$ Introduction de l'étude de la nature dans l'enseignement élémentaire et affermissement national, rénovation des curricula et des supports didactiques, enchantement et éthique de la nature, trois aspects de l'entrée de la nature dans les écoles primaires que nous proposons d'éclairer ici.

\section{Aux origines de l'étude de la nature dans les écoles primaires (années 1860-1880)}

6 Mais de quoi parle-t-on exactement à l'école primaire quand on utilise le terme de nature ? Pour circonscrire l'étendue de ce concept « instable », il convient d'en laisser monter le sens tel qu'il affleure sous la plume des élèves et des pédagogues. De cette aire de fabrication, il se dégage une conception partagée de la nature que le républicain-pédagogue Henri Marion formule ainsi : « on met en cause l'univers avec ses lois, le cours des choses, l'ensemble de tout ce qui est, vit et se meurt sous le soleil ».

${ }^{3}$ Déclinée en des termes très généraux, la proposition du professeur Marion reflète pourtant la perception de la nature véhiculée dans le corpus scolaire: non pas des espaces vierges débarrassés de tout aménagement, non pas des territoires soustraits à l'action de l'homme, mais une nature métissée combinant les éléments naturels et les facteurs humains. Une "nature ordinaire $»^{4}$ en quelque sorte, dans laquelle la biodiversité des jardins, des serres, des parcs zoologiques côtoie, dans la même réalité, les lieux non anthropisés des fronts pionniers. Et c'est précisément parce que les conceptions de la nature affectent des formes multiples, hybrides et constamment renégociées qu'il nous faut échapper aux vulgates disciplinaires pour instruire cette alliance entre l'enseignement primaire républicain et l'étude de la nature.

7 À quel moment, au juste, la nature s'insinue-t-elle dans les supports de l'enseignement primaire? Autrement dit, à partir de quand peut-on parler d'une étude de la nature dans les écoles élémentaires? Puisqu'il faut bien lui assigner une date précise, concédons que l'année 1867 pourrait constituer une sorte de moment initial. Non pas tant en raison de l'adoption des réformes du ministre Duruy que pour la vulgarisation de l'emblématique leçon de choses qui est d'abord une leçon sur les choses de la nature. En 1867, la directrice de l'École normale maternelle de Paris, Marie Pape Carpantier, 
donne, dans le cadre de l'Exposition Universelle, une série de conférences à la Sorbonne. Ses propos popularisent l'expression de leçon de choses auprès des instituteurs venus assister à ses conférences. La publicité faite alors à cette méthode venue des salles d'asile ${ }^{5}$ promeut l'étude de la nature dans l'enseignement primaire. Est-ce si sûr ? Oui, répond l'historien Pierre Kahn. De quelles choses auraient bien pu relever ces leçons, «si ce n'est de ces choses que les enfants peuvent observer, c'est-àdire d'abord les phénomènes de la nature et la transformation des objets par l'industrie humaine » (Kahn, 2002).

Mais à y regarder de près, cette date doit être davantage vue comme un repère commode plutôt que comme une rupture radicale. Car s'il prend forme dans ces années 1860 , le processus d'introduction de l'étude de la nature suit la pente des réformes voulues par les républicains-pédagogues. Préparée dans la décennie 1870, effective dès les années 1880, "l'effervescence pédagogique" (Chervel, 1988) transforme le quotidien de l'écolier : elle lui ouvre les portes d'un univers plus grand que tout ce qu'il avait pu imaginer. La nature qu'on lui met sous les yeux a beau avoir le goût du lointain, elle se garde bien de le délier de son environnement immédiat (Chanet, 1996). Véritable point d'orgue de cette pédagogie de terrain, les «classespromenades $»^{6}$ de l'inspecteur primaire Blanguernon sont lancées en 1909. En quatre décennies, c'est une véritable « révolution naturelle » qui a transformé les pratiques et les savoirs scolaires. Quel chemin parcouru depuis les véhémentes et utiles exhortations formulées par des scientifiques de renom dans les années 1870. Voici celle de l'influent Albert Dastre ${ }^{7}$, disciple de Claude Bernard, se livrant dans le Dictionnaire de pédagogie à un constat désolé :

Les vrais fondements de la science résident dans l'étude de la nature (...) Mais s'il en est ainsi (...) et si aujourd'hui les sciences de la nature ont remplacé comme instrument les sciences abstraites ou sciences métaphysiques, il semble qu'elles devraient avoir une part prépondérante dans l'éducation. Il n'en est rien. Jusqu'à ces dernières années, le préjugé mathématique a dominé, et les sciences abstraites ont eu la part léonine dans l'enseignement. Cette erreur serait funeste si elle se prolongeait trop longtemps.

Plus diplomate, et peut-être assuré de la réussite du plan d'éducation concocté par les républicains-pédagogues, voilà Augustin Boutan, le directeur de l'enseignement primaire entre 1873 et 1879 , se féliciter, dans l'article «expériences » du Dictionnaire, des évolutions en cours :

Comprend-on en effet qu'un enfant qui, de six à treize ans, a fréquenté assidûment l'école, ait été maintenu systématiquement étranger, par le genre d'études qui lui est imposé, au monde matériel qui l'entoure ? Comprend-on que les phénomènes dont il est journellement le témoin soient demeurés pour lui lettre morte ; qu'on ne lui ait jamais parlé qu'indirectement ou accidentellement des plantes, des animaux, avec lesquels il est sans cesse en contact ? (...) Pourquoi ne figurait-elle à aucun titre dans les programmes des écoles primaires? (...) Mais le point essentiel est acquis (...) le monde de la nature n'est plus un monde fermé pour les petits enfants. ${ }^{9}$

De fait, l'examen critique du curriculum scolaire est sans appel : dès les années 1870, la nature jaillit de toutes parts: manuels scolaires, réflexions des pédagogues, revues professionnelles proposent une multitude de documents s'intéressant à la nature. Ce nouveau paradigme éducatif traduit un renversement des positions traditionnelles. Jusqu'à fonder un monde meilleur ${ }^{10}$ ? Beaucoup le pensent. "L'ivresse scientifique $»^{11}$ des maîtres appuie le discours des autorités sur la connaissance des faits de nature. Mais lequel exactement ? Celui de Marcellin Berthelot, de Claude Bernard et 
de Paul Bert, celui-là même qui exhale des relents prononcés de positivisme ou bien celui d'un Henri Marion ou d'un Ferdinand Buisson se tenant fermement à distance des thuriféraires du tout rationnel? Hors de question, pour ces derniers, d'assécher un enseignement dont on attend certes qu'il émancipe les futurs citoyens, mais sans qu'il renonce à éveiller les émotions, les sensations et les sentiments des enfants. Pas une discipline n'échappa à l'impérialisme des "merveilles de la nature » que nombre d'éducateurs tenaient comme l'aiguillon de l'éducation pour tous. Prêter allégeance aux mystères de la nature et de l'univers plutôt que prétendre tout expliquer, tout éclairer, tout découvrir au prisme du rationalisme, c'était aussi la philosophie de Ferdinand Buisson, qui appelle les maîtres à initier leurs élèves à lever les yeux vers le ciel : «Ils ne l'ont jamais vu. Ils n'ont jamais été saisis de cette pensée des mondes innombrables, et de l'ordre éternel, et de l'éternel mouvement de l'univers. Éveillez-les à ces idées nouvelles, faites-leur apparaître ce spectacle de l'infini devant lequel se prosternaient les premiers pâtres de l'Asie et devant lequel tremblait le génie de Pascal » (Buisson, 1878).

11 En suivant le père de l'école laïque dans sa quête d'absolu, on peut deviner la tension qui sourde derrière les représentations de la nature dans la littérature scolaire: la concurrence - jamais tranchée - entre d'un côté une conception sécularisée, rationalisée, objectivée du savoir et de l'autre, une position traditionnelle, poétique et mystique de la nature qui se déplie aussi bien dans la littérature que dans les sciences. Pour le dire autrement, c'est en désordre que la troupe des pédagogues affectée au service de l'école primaire affronte l'idée de nature. Là, dans les sciences et dans l'agriculture, la tentation de forcer la nature, de la "soumettre à la torture $»^{12}$ coexistera avec une admiration émerveillée devant l'odyssée de quelques invertébrés microscopiques. Ici, dans la leçon de géographie ou dans le cours de français, le récit du combat de l'homme contre la nature voisinera avec une ode à la beauté des paysages nationaux. Ailleurs, dans le jardin scolaire ou dans les sentiers qui mènent à l'école, l'élève, tantôt apprendra à arracher à la nature ses meilleurs produits, tantôt se contentera d'en contempler les virtualités et de soulever délicatement le voile de ses secrets. Sous le large spectre des façons de dire la nature, émerge un faisceau de sensibilités si proches que l'on peut mettre au jour une vision commune ${ }^{13}$ de l'appréhension de la nature. La plus politique relève de la mystique nationale. Les éducateurs de tout rang se retrouvaient sur l'exigence de la passion de la France que la circulation des représentations de la nature était censée favoriser.

\section{L'affermissement national ou comment « refaire la France »}

Contre les divisions et les imperfections de la République naissante, l'école assuma la lourde tâche de "fabriquer des républicains ". La révolution laïque des années 1880 , l'établissement de la gratuité absolue de l'enseignement primaire dans les écoles publiques (loi du 16 juin 1881) ainsi que l'obligation scolaire des enfants de 6 à 13 ans (loi du 28 mars 1882) constituèrent des leviers puissants de l'unité nationale. Mais dans leur obsession de l'unité patriotique, les gouvernants entendaient forger, chez les enfants, une passion profonde de la France. Refonder l'école pour asseoir le nouveau régime républicain, l'idée était déjà toute entière dans l'enseignement d'Edgar Quinet (Quinet, 1850). Mise en œuvre par Buisson et Guillaume, l'une de ses justifications 
fortes repose sur la conviction que l'histoire et la morale ne peuvent être l'unique clef de la construction nationale. Compte tenu des flétrissures du roman national, il pouvait être opportun de peindre une "doulce France". D'enseigner au fond une nation comblée par les prodigalités de la nature. Cette naturalisation de la France avait l'avantage d'éloigner le spectre de la division. Quoi de plus fédérateur qu'une éducation reposant sur «ce qui est à tout le monde $»^{14}$, s'appuyant sur des sensations que l'on expérimente individuellement, mais que l'on peut partager avec tous?

De sorte que la France imaginée par les républicains ne cessa de déployer ses charmes pour conquérir les écoliers. S'interrogeant sur la construction des identités nationales, Anne-Marie Thiesse (1997) et Jean-François Chanet (1996) ont démontré qu'une dialectique habile entre l'amour du pays natal et la grandeur de la patrie visait à affermir le sentiment national. Dans la littérature scolaire, toujours la même cantilène d'une France gâtée par la nature : « le même hymne : beauté et diversité ». Pas trop vite cependant. On se tromperait lourdement à vouloir enfermer l'étude de la nature dans la citadelle agronomique. Ni réduites aux figures agricoles, ni enfermées dans le pittoresque, encore moins circonscrites aux registres de la science, les figures de la nature composent une palette aussi originale qu'éclectique. D'où l'étalage de planches didactiques, d'images et de textes mettant en scène la profusion des espèces, l'étrangeté des paysages tropicaux, le récit intarissable des formes biologiques. Face à la démesure de la nature, les maîtres, fidèles en cela à l'esprit des programmes, préférèrent se consacrer à l'étude des terroirs, quitte à articuler les particularismes locaux avec l'universalité du monde. Et l'on comprend pourquoi.

En convoquant la nature pour affermir la construction nationale, le discours scolaire ne pouvait faire autrement que d'en restituer une image exaltante. À elle de maintenir le goût de la France et de cimenter le cœur des petits Français. À elle d'attacher les enfants à leur pays natal, de leur suggérer que les solidarités communales valent bien les promesses incertaines de l'exil vers la ville. Avec pareil entêtement bucolique se construisit le topique d'une France adossée à une nature bienfaisante. Ainsi, chemin faisant, la France que découvrent les deux orphelins du Tour de la France par deux enfants apparaît comme un pays de cocagne: accueillante, prospère, jalonnée de fermes modèles. Partout dans les supports scolaires, les potentialités naturelles ont débouché sur une épiphanie heureuse de la ruralité. Pour les autorités, c'était un moyen de garantir la vitalité d'un lien national vissé à l'étude de la nature. De quoi, au fond, réenchanter un pays confronté à la montée de l'individualisme et menacé par l'effritement des solidarités traditionnelles. De quoi aussi formuler un prêt à penser pour justifier «l'appendice colonial»: en l'espèce, quelque chose comme des terres prodigieuses où se dressait une marqueterie affligeante d'espaces naturels inexploités et saccagés par la brutalité des indigènes. De sorte que, dans ces «terres sauvages », la diffusion du modèle français permettait de « libérer » les populations de la tyrannie de la nature (Bonneuil, 1997). ${ }^{15}$ Pour être prioritaire, l'entreprise de nationalisation des esprits ne visait pas à assigner les élèves à résidence et encore moins à les enfermer dans une vision étriquée de leur environnement naturel. Cependant, les larges espaces d'expression et de réflexion sur «l'éducation à la nature » dépassent de très loin, les seuls enjeux politiques. 


\section{Rénover les curricula pour enseigner autrement}

En empruntant ses déclinaisons aux formes géographiques, littéraires ou scientifiques, la diversité des représentations de la nature a ouvert la voie à de nouvelles pratiques, et en particulier à l'instauration d'une pédagogie renouvelée. Ni tout à fait neuves, ni tout à fait assumées par l'Institution scolaire, ces méthodes d'enseignement ont lentement conquis une partie des écoles de la République. Ni tout à fait neuves, elles ont pour la plupart été recommandées et théorisées par les précurseurs de l'Éducation nouvelle (Rousseau, Pestalozzi, Frœbel...). Ni tout à fait assumées, car une majorité de maîtres proteste contre des innovations auxquelles ils n'ont pas été préparés. Mais soyons clair, derrière les initiatives remarquables de beaucoup d'entre eux, il y a, au fond, la conviction profonde que l'écolier est d'abord un enfant. «Ne pas tuer l'enfant dans l'écolier ${ }^{16}$, la recommandation du $\mathrm{D}^{\mathrm{r}}$ Élie Pécaut résume l'essence même de la modernité éducative dont les principes sont déroulés dans le Dictionnaire de pédagogie: ce porte-voix de l'école républicaine. À lire les développements de Pécaut sur l'ennui, sur la poésie, à parcourir les notices sur les promenades, l'astronomie, la géologie, à suivre Ferdinand Buisson et James Guillaume évoquer les mondes de la nature, on ne peut s'empêcher de penser que ces pédagogues rêvaient une école différente. Car ils ne se contentent pas de nouer la gerbe des mots, des idées et des intentions qui les animent, ils tracent une perspective en invitant à rompre avec des méthodes et des usages surannés, quitte à bousculer les autorités constituées.

Et les voix qui résonnent ici se fondent dans le répertoire infini de l'attention à l'enfant. Cette chorale est un véritable traité de l'épanouissement des écoliers avec, en toile de fond, l'idée sincère que l'enfance se confond étroitement avec "le temps du rêve ». Ignorer ce tendre glacis éducatif, c'est prendre le risque de ne rien comprendre au jaillissement de la nature dans les écoles de la Troisième République. Car rien ne serait plus faux que de réduire l'éducation à la nature à l'obsession patriotique. Et justement, c'est une tout autre dimension que l'on traverse en scrutant les activités menées d'une main de maître dans les écoles attentives à la rénovation pédagogique. La marée discursive sur la nature qui gagne toute la littérature scolaire atteste que ces pratiques n'ont pas été circonscrites à de rares écoles en dépit des maigres effectifs de pédagogues téméraires. Leçons de choses, leçons de sciences, leçons d'agronomie, leçons de géographie, leçons de mathématiques, toutes pouvaient être prétexte à la mise en spectacle de la nature. Trop souvent, sans doute, l'instituteur était seul en scène. On peut regretter là une certaine conception spéculaire du savoir, à l'évidence contraire à l'esprit de l'école moderne voulue par les républicains. Il n'empêche, la "révolution naturelle» était en marche. Inexorablement. Le mouvement de transformation de la classe par de nouvelles pratiques ne pouvait plus s'abriter sous la toile légère de quelques instituteurs intrépides. Évidemment tous les maîtres ne rêvaient pas nécessairement de la même école républicaine; il ne faudrait pas pour autant rabattre les innovations introduites par les maitres, à de minces correctifs d'une pédagogie jugée rébarbative.

17 À y regarder de près, on croit changer d'école. Jusqu'à quel point au juste ? À dire vrai, tout dépendait du maître. Et dans son vigoureux effort pour s'élever au-dessus des routinières dévotions à la tradition ${ }^{17}$, une poignée a eu l'immense mérite d'éveiller les esprits engourdis par le calme morne de la classe. Ils ont dû pour cela recourir quotidiennement aux méthodes actives, mobiliser les sens de toutes ces «âmes 
tendres » et développer l'enseignement par l'aspect. ${ }^{18}$ Encore ne faudrait-il pas négliger les incertaines tentatives pour transporter des enfants pas si prompts que cela à s'émerveiller des audaces de leur maître! (Jakez Hélias, 1977). Cela posé, n'est-il pas un peu exagéré de penser que l'étude de la nature a constitué le fer de lance de cette effervescence éducative ? Puisque l'on a puisé dans les travaux de Mona Ozouf, c'est à elle que nous confions la principale objection à cette interrogation:

À la communale, on fait écrire aux élèves, quand ils arrivent en classe, le temps qu'il fait, si le coucou a chanté, si la première ficaire est apparue sur un talus ensoleillé. L'air du dehors pénètre, on apporte à la maîtresse, trois châtaignes, un bouquet de narcisses. C'est une école ouverte sur la nature ${ }^{19}$.

S'il n'a pas échappé à la grande historienne que l'école primaire consacrait autant d'allant à la nature, c'est bien parce que se sont imposées dans les classes mille façons de connaître, d'étudier, de regarder et d'apprécier les plantes, les animaux et toutes les compositions paysagères que l'on soumettait à l'appréciation des sens des enfants. Par le biais d'un dialogue ouvert entre les sciences, l'art, la géographie, la littérature ou le dessin - sorte d'antichambre de l'interdisciplinarité - l'école rénovée agença une espèce de laboratoire dédiée aux choses de la nature. À l'antique leçon de mots, parfois appuyée par d'illustres gravures, vinrent, par exemple, s'ajouter - parfois se substituer - une lente fabrique de musées scolaires et des minutieuses confections d'herbiers. On touche ici à deux exemples de la mutation opérée dans les classes aussi bien sur le plan didactique que sur le plan matériel. Trois en comptabilisant l'implantation des jardins dans de nombreuses écoles primaires. On aurait tort de n'y voir qu'une disposition réglementaire du plus bel effet décoratif. Car, disons-le tout net, entrés avec effraction dans les écoles, jardins, musées scolaires et herbiers présagent de la « mort lente » de la classe d'hier ${ }^{20}$.

Que de trouvailles ils exigent, que de besogne ils mobilisent, que de ruptures ils entrainent. "Allez debout» croit-on entendre de la bouche du maître. "Dehors les enfants » pourrait-on ajouter. Quelle que soit l'injonction que le maitre formule, elle commande de nouvelles attitudes face au travail, elle implique des activités nouvelles. Arpentage, amendements, bêchage, sarclage, récoltes ne sont pas très nouveaux pour des enfants de la campagne. Mais où trouver les échantillons de plantes endémiques, où ramasser les pierres insolites que l'on aime faire miroiter derrière les vitrines d'un petit meuble? Où capturer les malheureux insectes épinglés en rangs soigneusement alignés, sinon dans les alentours de l'école? Sous trois appellations distinctes, une même exigence de mouvement, une même mise en demeure d'activités. Car les musées et les herbiers - moins pour les activités de jardinage dont la parcelle jouxtait l'école subordonnent leur réalisation à l'ouverture sur l'extérieur, aux fameuses «classespromenades » dont l'inspecteur Blanguernon (1918) a théorisé les vertus. Et leur liste est longue. Il y a celles qui éveillent les esprits et forment les corps, celles qui font comprendre la géographie et la géologie, puis celles qui suscitent l'imagination et inspirent les poètes et les peintres et celles qui guident la main lors des rédactions ; mais aussi celles qui facilitent le relevé des cotes de l'arpentage et celles, enfin, qui guident la collecte des trésors naturels que l'on étudiera en classe. Et d'abord dans la leçon de choses, cette devanture officielle de l'école active. Sous l'œil du maître, l'écolier doit s'y livrer aux observations et aux expériences qui font de lui une espèce de savant en miniature. Idéalement, car le plus souvent la tâche de l'élève se limitait à rester attentif aux manipulations méticuleuses de son instituteur. Mais si les enfants se contentent d'observer le maître procéder à l'expérimentation, la confection des 
herbiers et des musées ou le travail au jardin leur offrent l'occasion de mettre « la main à la pâte ${ }^{21}$

$20 \mathrm{Au}$ rendez-vous scolaire de toutes les productions sur la nature, les écheveaux du nouvel arsenal pédagogique tissent des savoirs jusque-là ignorés. Petite chambre des merveilles, les échantillons du musée scolaire symbolisent les besoins d'une pédagogie réinventée. Non pas d'ailleurs une nature mise sous cloche, et figée pour des décennies, puisque la fabrique du musée s'inscrivait dans un processus continu de renouvellement. Ferdinand Buisson ne manqua pas de le rappeler: "Il serait à souhaiter que chaque génération scolaire eût à le reconstituer; car, le grand profit à tirer de ces petits musées, ce n'est pas de les avoir, c'est de les faire $»^{22}$. Désormais, sur une table, dans une armoire, ou sur les murs d'une salle de classe, sont entreposés et exposés des objets hétéroclites: squelettes, pierres taillées, insectes variés, décoction de plantes voisinant avec des fioles insolites, pipettes, instruments scientifiques, images naturalistes et cartes géographiques.

21 Il existe, dans la mise en scène de tous ces échantillons une esthétisation du végétal, une théâtralisation qui demeurent un levier incontestable de questionnements et d'émotions: émerveillement, étonnement, enthousiasme naissent de cette confrontation à un vivant embaumé. Nés des pratiques de terrain partout reproductibles, musées et herbiers font ainsi la démonstration que la connaissance du monde s'inscrit dans une connaissance intime de l'espace de l'école, de son environnement et de la nature avec laquelle on commerce. S'ébauche avec ce type de démarche, l'étude des relations entre les êtres vivants. Conquérant, ce nouveau régime de scientificité a autorisé de nouvelles perceptions de l'environnement participant ainsi à l'élaboration de représentations en rupture avec les affects traditionnels. Puisque les sens des enfants étaient mis à l'épreuve d'une nature revisitée, ils regardèrent autrement le monde qu'ils avaient toujours eu sous les yeux. Mieux, les manières de le dire et de le raconter esquissent au hasard du dialogue entre sciences, arts, littératures et « enseignement dehors » ce qui ressemble fort sinon à une éthique au moins à l'éclosion du sentiment de la nature.

\section{Enchanter et respecter la nature}

Pour qui voudrait prouver que l'école de la Troisième République a épousé les préoccupations technoscientifiques de son temps, il serait possible de ressusciter les exercices scolaires invitant à tout compter et décompter, à tout mesurer, à tout peser, à tout énumérer, à tout classer, à tout ordonner et... à tout démystifier. Il serait possible de décrire par le détail toutes les mentions des programmes scolaires visant à faire advenir un Prométhée moderne suffisamment habile pour dérober à la nature ses secrets intimes tout en étant capable de triompher des forces telluriques et assez expérimenté pour ployer les humeurs du ciel sous le joug du progrès. Jusqu'à démontrer que l'école était porteuse d'un projet rationaliste aux saveurs de son temps. Pourquoi pas ? Routes, ponts, hygiène publique et privée, pasteurisation et vaccination, animaux nuisibles, modernisation agricole, machinisme, industrialisation pimentent tous ces ouvrages livrés à la curiosité des maîtres et des enfants. Rarement interrogée ou alors si discrètement, l'idée de domestication de la nature a longtemps éclipsé les explorations discordantes des chercheurs de l'histoire environnementale. Au point que l'on en a oublié la dimension affective de l'éducation à la nature, passée sous silence ou 
réifiée par une historiographie interrogeant la modernité éducative à l'aune du progrès industriel. En martelant le conservatisme des pratiques scolaires, en faisant des salles de classe le réceptacle d'une société paysanne archaïque, en psalmodiant la trilogie du «lire, écrire, compter ", en se détournant de l'enseignement informel - entendons nondisciplinaire - la mémoire collective a négligé l'une des caractéristiques centrales du projet des républicains-pédagogues, à savoir celle d'une éducation soucieuse d'équilibre, de respect et, plus encore, d'harmonie entre les individus et leur milieu naturel. Une école, finalement, en avance sur son temps et davantage préoccupée d'apprentissages sensibles que d'académisme ou de conservatisme. Car enfin, c'est par une telle préoccupation, celle d'enchanter le quotidien des classes sans prendre le risque de délier les enfants de leur milieu natal, que les autorités scolaires ont engagé, en misant sur l'éducation à la nature, un corps à corps vigoureux avec les philosophies d'inspiration cartésienne et positiviste. Suffisant, c'est certain, pour imposer des savoirs susceptibles d'inspirer aux enfants une passion raisonnable pour les choses de la nature. Et encore! Les tenants de l'éducation intégrale ont poussé plus loin la lutte sur les enjeux d'une éducation naturaliste. ${ }^{23}$ Il n'y a d'ailleurs pas qu'eux. Des scientifiques de renom, des psychologues, des écrivains, des politiques se sont joints à la cohorte des maîtres avisés pour éviter que la nature ne soit réduite à un pur objet d'étude, à une simple occurrence ou pire encore à une triviale ressource réservée à l'entier bénéfice de celui qui sait l'exploiter. Des noms ? Les géographes Élisée Reclus et son cousin Schrader, les historiens Michelet et Quinet, les scientifiques Perrier et Flammarion, les Pécaut père et fils, puis encore la cheville ouvrière du Dictionnaire de pédagogie James Guillaume, les politiques Ferry et Jaurès, autant de personnalités accordées face la complexité de la biodiversité et qui racontèrent leurs épanchements devant le spectacle de la nature. Peut-être plus éclairantes encore, les subtiles confessions naturalistes de Ferdinand Buisson, «le père de la laïque » auxquelles on peut joindre, pour leur exemplarité, les analyses de son successeur, l'éminent psychologue Henri Marion sur les relations entre l'homme et la nature. Il faut s'y arrêter.

En suivant Ferdinand Buisson jusqu'à l'article « lyrique » du Dictionnaire de pédagogie, on prend note de ce que la culture poétique de la nature doit encore aux grands écrivains du XIX ${ }^{e}$ siècle (Buisson, 1887). Avec cette anthologie de la poésie française proposée aux instituteurs, les éducateurs républicains professent leur attachement à la contemplation de la nature qui reste, à leurs yeux, une des conditions essentielles de la "grandeur d'âme". Mais sur le terrain, quelle démarche adopter? Par une initiation graduelle prévue sur toute la scolarité, l'esprit de l'enfant se pénétrait lentement des harmonies de la nature. L'écoute attentive des «battements de son cœur » démarre dès le cours élémentaire par la lecture de textes simplifiés. Elle se poursuit au cours moyen par des textes plus complexes, mais dont la tonalité reste fortement marquée par l'agrarisme. Aux côtés des auteurs contemporains (Rendu, Autran, Aicard, etc.), les grands écrivains (Chateaubriand, Lamartine, Hugo, etc.) réussissent à se frayer un chemin dans les manuels les plus vendus. Livrés à la curiosité des écoliers, les extraits des grands auteurs empruntent encore au bonheur des champs et ne renvoient pas directement au Sublime. Il s'agit plus de glorifier la «belle campagne » que de favoriser les épanchements de l'âme. Les semailles, les fenaisons, le retour des troupeaux, les paysages bocagers voilent les mystères de la nature, retardent l'éclosion d'un authentique sentiment de la nature. Une fois ce préavis expiré, le lyrisme, le sublime et le vertige de la nature emplissent littéraire (romanesque, poétique ou autre), la 
perception esthétique de la nature génère une dimension affective, émotionnelle qui introduit ou renforce le lien entre les enfants et la nature. N'allons pas imaginer que cet enchantement ne serait qu'une vaine espérance de pédagogues idéalistes et que la poésie de la nature n'aurait d'autres vertus que celles d'un exercice d'expression théorique. Les analyses d'Henri Marion tordent le cou à ces supputations.

L'homme appartient au collectif d'intellectuels républicains dont l'action et la réflexion pédagogique ont pour longtemps assigné à l'enseignement primaire son organisation et ses missions. De la nature, il pense qu'elle est « un artiste incomparable » dont les œuvres inégalables "comblent les hommes d'admiration" (Marion, 1882). Comme psychologue, il se rallie à l'idée que » la contemplation de la nature est donc propre à nous rendre modestes » (Marion, 1884). Attention néanmoins. Cette admiration, Marion l'inscrit au rang des devoirs et la lie à l'obligation de respect que les individus doivent à l'égard de l'universel :

Si les sentiments se commandaient, si ceux de l'ordre esthétique et poétique, en particulier, ne supposaient pas une culture qui nécessairement fait souvent défaut, il ne faudrait pas hésiter à déclarer que c'est un devoir pour tout homme, de contempler avec respect la nature, et d'éprouver pour elle les sentiments qu'elle inspire toujours à qui sait la regarder (Marion, 1884).

À l'idée que l'esthétique naturelle nourrit le sentiment de la nature, il superpose le principe d'une culture préalable pour goûter cette émotion. L'esthétique qu'il décrit est donc de l'ordre constructiviste et cognitiviste. Mais le spécialiste de l'enseignement de la morale va plus loin encore. Cette sympathie avec la nature, elle n'est pas simplement une posture valorisante. Non, elle implique un devoir de bonté envers les vivants, aussi bien les plantes que les animaux. Et c'est bien parce qu'il est conscient "que nous sommes contre eux en état de guerre perpétuelle» les pages des ouvrages du cours supérieur. ${ }^{24} \mathrm{Ce}$ qui n'exclut pas, expliquait Buisson, un contact plus sensuel, mais pas moins émerveillé avec la nature. Peu importe, au fond, son expression littéraire (romanesque, poétique ou autre), la perception esthétique de la nature génère une dimension affective, émotionnelle qui introduit ou renforce le lien entre les enfants et la nature. N'allons pas imaginer que cet enchantement ne serait qu'une vaine espérance de pédagogues idéalistes et que la poésie de la nature n'aurait d'autres vertus que celles d'un exercice d'expression théorique. Les analyses d'Henri Marion tordent le cou à ces supputations.

L'homme appartient au collectif d'intellectuels républicains dont l'action et la réflexion pédagogique ont pour longtemps assigné à l'enseignement primaire son organisation et ses missions. De la nature, il pense qu'elle est "un artiste incomparable » dont les œuvres inégalables "comblent les hommes d'admiration" (Marion, 1882). Comme psychologue, il se rallie à l'idée que » la contemplation de la nature est donc propre à nous rendre modestes » (Marion, 1884). Attention néanmoins. Cette admiration, Marion l'inscrit au rang des devoirs et la lie à l'obligation de respect que les individus doivent à l'égard de l'universel :

Si les sentiments se commandaient, si ceux de l'ordre esthétique et poétique, en particulier, ne supposaient pas une culture qui nécessairement fait souvent défaut, il ne faudrait pas hésiter à déclarer que c'est un devoir pour tout homme, de contempler avec respect la nature, et d'éprouver pour elle les sentiments qu'elle inspire toujours à qui sait la regarder (Marion, 1884).

27 À l'idée que l'esthétique naturelle nourrit le sentiment de la nature, il superpose le principe d'une culture préalable pour goûter cette émotion. L'esthétique qu'il décrit est 
donc de l'ordre constructiviste et cognitiviste. Mais le spécialiste de l'enseignement de la morale va plus loin encore. Cette sympathie avec la nature, elle n'est pas simplement une posture valorisante. Non, elle implique un devoir de bonté envers les vivants, aussi bien les plantes que les animaux. Et c'est bien parce qu'il est conscient "que nous sommes contre eux en état de guerre perpétuelle » qu'Henri Marion pense qu'il faut reconnaître aux animaux des droits au même titre que les hommes avec lesquels ils partagent " une étincelle de la vie universelle, un rayon de la raison divine » (Marion, 1884). Sans faire des animaux des bénéficiaires de droits à part entière, les injonctions de Marion vont au-delà des strictes recommandations de sauvegarde et de protection du patrimoine animal - ce qui le rangerait déjà parmi les avant-gardistes de cette cause - puisqu'il conçoit une relation équilibrée entre les hommes et les autres espèces : les hommes partageant en quelque sorte les territoires et leurs ressources. Pour Marion, c'est au maitre qu'il revient d'inculquer à ses élèves des habitudes de douceur et de bonté envers les animaux. Plus encore. Non seulement il doit leur apprendre à veiller à la conservation de certaines espèces - comme les moineaux domestiques habituellement pourchassés sans pitié -, mais il doit encore leur apprendre à éloigner les dangers qui les menacent. À la classique séparation entre nuisibles et utiles, Marion substitue celle d'un monde naturel commun, celle d'une nature en partage qui impliquent d'amener les écoliers «à comprendre la nature et à l'aimer ». Cette conscience de l'interdépendance des destins animal et humain, loin de triompher, circule lentement dans les méandres tortueux des discours scolaires. Combien sont-ils les auteurs qui militent pour que les enfants apprennent à aimer et à respecter les plantes et les animaux? Suffisamment pour qu'une culture "écologique » infuse lentement et inocule un sentiment d'attention à l'égard du vivant. Il est certain que tous les penseurs de l'école républicaine ne poussèrent pas la réflexion aussi loin que Marion, mais les appels répétés à la bonté et au traitement amical des animaux reflètent la montée d'une véritable empathie pour les espèces proches de l'homme, ou supposées telles. En d'autres termes, dans les écoles de la République, la question du vivant n'est plus posée seulement en termes d'utilité, mais elle consigne le problème de la valeur intrinsèque des êtres naturels indépendamment de leur relation à l'homme. En soutenant «que nous ne sommes pas isolés dans la nature » et « que des liens étroits nous unissent à tous les êtres " (Marion, 1884), la morale laïque défendue par Henri Marion inculque aux enfants une nouvelle éthique comportementale tout en diffusant une conception de l'univers éloignée de l'arrogance technoscientifique.

Un mot encore. Poussée par les pédagogues et les savants de son temps, l'école primaire a lentement construit, à partir des années 1870 , une pensée de la nature nourrie de curiosités insatiables et d'attentions constamment renouvelées de la part des maîtres et des élèves. Jamais de mépris pour les œuvres de la nature, mais une passion pour les êtres qui l'animent, auxquels se joignent les ruses de la pédagogie pour faire aimer et respecter tout ce qui la compose. Voilà posées les bases d'un séminaire qui semble revenir au goût du jour. 


\section{BIBLIOGRAPHIE}

Bataille, F. (1899). Lectures françaises illustrées de l'école. Cours élémentaire. Paris : A. Lemerre. Blanguernon, E. (1918). Pour l'école vivante. Paris : Hachette.

Bonneuil, C. (1997). Mettre en ordre et discipliner les tropiques : Les sciences du végétal dans l'empire français, 1870-1940, Doctorat d'histoire des sciences. Université Paris-Diderot - Paris VII. Bruno, G. (1877). Le Tour de la France par deux enfants. Devoir et patrie : livre de lecture courante avec 200 gravures instructives pour leçons de choses, Paris : E. Belin. (réédit.1977)

Buisson, F. (1880). Conférence sur l'enseignement intuitif du 31 août 1878. Dans Les conférences pédagogiques faites aux instituteurs délégués à l'Exposition universelle de 1878 Paris : Delagrave. Buisson, F. (dir.) (1882-1887), Dictionnaire de pédagogie et d'instruction primaire. Paris : Hachette, 4 volumes.

Cabanel, P. (2007). Le Tour de la nation par des enfants. Romans scolaires et espaces nationaux (XIX ${ }^{\mathrm{e}}-\mathrm{XX}^{\mathrm{e}}$ siècles). Paris : Belin.

Chanet, J.-F. (1996). L’École républicaine et les petites patries. Paris : Aubier.

Chervel, A. (1888). L'histoire des disciplines scolaires. Réflexions sur un domaine de recherche, Histoire de l'éducation, $n^{\circ} 38$, p. 59-119.

Chevalier, J.P. (2003). Du côté de la géographie scolaire. Matériaux pour une épistémologie et une histoire de l'enseignement de la géographie à l'école primaire en France, HDR. Université Panthéon-Sorbonne - Paris I. Consulté sur https://tel.archives-ouvertes.fr/tel-00218343v2/ document

Crubellier, M. (1993). L'école républicaine 1870-1940. Esquisse d'une histoire culturelle. Paris : Editions Christian.

Dasi, P. (2019). L'école primaire et le récit colonial sur la nature (1870-1914). Le Télémaque, 2019/2, nº 56, p. 143 à 156.

Dasi, P. (2021). Penser et représenter la nature à l'école sous la Troisième République. Paris : L'Harmattan.

Jakez Hélias, P. (1977). Le cheval d'orgueil. Mémoires d'un breton du pays bigouden. Evreux : Plon.

Kahn, P. (2002). La leçon de choses. Naissance de l'enseignement des sciences à l'école primaire. Villeneuve-d'Ascq : Presses universitaires du Septentrion.

Kahn, P. Leçons de choses et enseignement des sciences en France à la fin du 19e siècle : contribution à une histoire de la culture scolaire. Hist.Educ. Porto Alegre, 2014, p. 183-201.

Marion, H. (1884). Leçons de morale. Paris : Armand Colin.

Marion, H. (1882). Leçons de psychologie appliquées à l'éducation. Paris : Armand Colin.

Ozouf, J. et M. (1992). La République des instituteurs. Paris : Gallimard/le Seuil.

Quinet, E. (1850). L'enseignement du peuple. Paris : Chamerot Libraire.

Quinet, E. (1870). La Création. Paris : Librairie internationale. 
Reclus, E. (1866). Du sentiment de la nature dans les sociétés modernes. Revue des deux mondes, $\mathrm{n}^{\circ} 63$, p. 352-381.

Thabault, R. (1944). 1848-1914. L'ascension d'un peuple. Mon village. Ses hommes, ses routes, son école. Paris : Delagrave.

Thiesse, A.-M. (1997). Ils apprenaient la France : l'exaltation des régions dans le discours patriotique. Paris : Éditions de la Maison des Sciences de l'Homme.

\section{NOTES}

1. Voir également Pierre Dasi (2021).

2. Il a fallu tout le génie des maîtres pour articuler ces deux ambitions en tension. Le projet patriotique s'accordait, lui, aisément avec l'ordre scolaire traditionnel tandis que l'Éducation nouvelle supposait de rompre avec les pratiques habituelles.

3. Il a fallu tout le génie des maîtres pour articuler ces deux ambitions en tension. Le projet patriotique s'accordait, lui, aisément avec l'ordre scolaire traditionnel tandis que l'Éducation nouvelle supposait de rompre avec les pratiques habituelles.

4. Nous empruntons ce concept à la pensée environnementale de la fin du $\mathrm{XX}^{\mathrm{e}}$ siècle. Il renvoie à une réalité plus complexe que celle que donnait à penser le partage binaire entre les espaces naturels et artificiels. La nature ordinaire comprend une mosaïque d'espaces naturels plus ou moins façonnés par l'homme qui vont des champs cultivés à la « wilderness » étatsunienne. Lire Rémi BEAU (2015). « Nature (ordinaire)». Dans Dominique Bourg et Alain Papaux (dir.). (2015). Dictionnaire de la pensée écologique. Paris : PUF, coll. « Quadrige », 2015, p. 685-688.

5. Asile : c'est ainsi que l'on appelait les établissements destinés à accueillir les petits de deux à six ans, qui ont précédé jusqu'en 1881 les écoles maternelles.

6. Le syntagme apparaît, en 1909, dans une circulaire de l'Inspecteur d'académie de la HauteMarne Edmond-Désiré Blanguernon. Cette appellation connaît un vif succès et est commentée dans de nombreuses revues professionnelles et en particulier dans le Manuel général.

7. Successeur de Paul Bert à la chaire de physiologie de la faculté des sciences de Paris à partir de 1876, cet agrégé de physique rédige l'article «Science » du Dictionnaire de pédagogie et d'instruction (DP1 et DP2)

8. Albert Dastre, (1882), article « Science », DP2, p. 2006

9. Augustin Boutan, (1882), article «Expériences » DP1, p. 972. Il est évident que le monde de la nature n'est pas étranger aux écoliers - enfants des campagnes très majoritairement. Pour Boutan, il s'agit de soumettre les représentations des enfants à l'épreuve d'un enseignement positif.

10. «Par-delà la mort et le tombeau, nous appelons un monde meilleur, des vies plus élevées, des formes plus belles, des êtres plus achevés [...]. Je ne voudrais pas borner cette croyance à n'être que la vision anticipée des développements de la vie à travers des âges futurs géologiques ; il est certain que dans cet instinct d'un monde meilleur, je trouve la loi qui est aujourd'hui révélée, publiée, manifestée par la science de la nature». (Quinet, 1868).

11. "Et nous participions à cette ivresse scientifique qui était celle d'une génération qui avait lu Taine et Renan (et lisait Haeckel et Le Dantec) sur la vie matérielle transformée par les applications des sciences physiques, la médecine rénovée par les sciences biologiques ». Cité par Mona Ozouf dans La République des instituteurs.

12. Cité par Pierre Hadot (2004). Le voile d'Isis. Essai sur l'histoire de l'idée de nature. Paris: Gallimard, «Folio-essais », p. 167.

13. C'est bien l'une des originalités de l'école primaire que d'avoir sécrété un mariage hétéroclite entre des sensibilités philosophiques variées (positivisme, naturalisme, romantisme, 
spiritualisme, kantisme...) et les représentations de la nature qui parcourent la littérature scolaire.

14. «Non réellement, il faut mieux parler d'autre chose, de ce qui est à tout le monde, du soleil, de la lune, des étoiles, des saisons, des nombres, du fleuve, de la montagne, de façon que celui qui n'a point de chaussettes se sente tout de même citoyen; de façon que la maison d'école soit le temple de la justice, et le seul lieu où les pauvres ne soient pas méprisés ». Alain (1920). Les propos. Paris : Éditions de la nouvelle Revue Française.

15. À ce sujet, voir l'article de Pierre Dasi (2019).

16. Elie Pécaut, article « Hygiène scolaire », DP1, p. 1307.

17. Pour les contempteurs de l'enseignement traditionnel, la persistance d'une forme de scolastique, le principe récitatif, l'obsession de l'autorité, la docilité et «l'immobilisme» des enfants - le tout recouvert de passivité - caractérisaient le fonctionnement de la majorité des écoles primaires en France au XIX ${ }^{\circ}$ siècle.

18. À partir des années 1880 , cette expression vient éclairer et parfois se substituer à celle de Leçons de choses. Il vise à mobiliser les facultés perceptives, réflexives et même physiques de l'enfant. L'idée est de former le jugement de l'enfant en sollicitant, chez lui, toute une série d'opérations intellectuelles. Au fond, il s'agit de la mise en place des fameuses méthodes actives.

19. Mona Ozouf évoque ici l'école des années 1930, mais ces propos viennent confirmer toute l'entreprise accomplie depuis les débuts de la Troisième république. Ozouf $\mathrm{M}$. Conversation avec Mona Ozouf . Zadig, $\mathrm{n}^{\circ} 1$, p. 16.

20. La plupart des instituteurs n'étaient pas préparés à ces pratiques pédagogiques et beaucoup l'exprimèrent auprès de leur inspecteur. D'un autre côté, plusieurs décennies furent nécessaires pour que ces innovations ne se généralisent à toutes les écoles. Et bien souvent, elles n'occupèrent qu'une faible place dans les emplois du temps hebdomadaires.

21. On pense au laboratoire d'idées La main à la pâte, fondé en 1995, qui diffuse des pratiques innovantes pour améliorer la qualité de l'enseignement des sciences à l'école et au collège. Il s'agit de faire découvrir aux élèves une science vivante et accessible, favorisant par des pédagogies actives la compréhension des grands enjeux du 21e siècle, le vivre ensemble et l'égalité des chances.

22. Cité dans S.G. (1900) Guide pratique pour la composition et l'installation des musées scolaires. Paris : X. Rondelet et cie, Éditeurs, p. 5.

23. On pense à Paul Robin à Cempuis dans les années 1890 ou plus tard, à Célestin Freinet dans son école du Pioulier à Vence. Mais, en réalité, c'est toute l'Éducation nouvelle qui s'est saisie de ce paradigme éducatif.

24. Deux articles du Dictionnaire de pédagogie précisent les enjeux de la poésie. Félix Pécaut, « Poésie », DP1, p. 2387-2391 et Eugène Manuel, « Poésie », DP2, p. 1630-1634.

\section{RÉSUMÉS}

L'une des missions fondamentales de l'école de la Troisième République fut d'être l'institutrice de la nation. Mais comment procéder pour que chaque écolier ait «la patrie au cœur »? Dès 1867, l'idée neuve apportée par les philosophes-pédagogues, pour faire la France, n'était pas d'enseigner l'histoire ou la morale ; c'était d'apprendre la nature. D'où la lancinante cantilène du «miracle naturel de la France » dont l'écho a résonné dans toute la littérature scolaire jusqu'au 
$\mathrm{XX}^{\mathrm{e}}$ siècle. En prenant vigoureusement appui sur l'étude de la nature, les contenus disciplinaires bénéficièrent d'une opportune cure de jouvence que le renouvellement des méthodes pédagogiques - promenades, jardins, herbiers, leçons de choses - a accompagnée. Chose plus inattendue, en s'installant dans les salles de classe, l'éducation à la nature a fait émerger ce que l'on peut appeler une « éthique de la nature ».

One of the fundamental missions of the school of the Third Republic was to be the teacher of the nation. But how can we ensure that each schoolchild has "the homeland at heart" ? As early as 1867 , the new idea brought by philosophers and teachers to make France was not to teach history or morality; it was to learn about nature. Hence the haunting cantilena of "the greatness of France", which resonated throughout school literature until the 20th century. However, by vigorously relying on the study of nature, the disciplinary contents benefited from a well-timed rejuvenation that was accompanied by the renewal of pedagogical methods - walks, gardens, herbaria, objects lessons -. More unexpectedly, by moving into the classroom, nature education has led to the emergence of what can be called an "ethics of nature ".

\section{INDEX}

Mots-clés : innovation pédagogique, enseignement primaire, histoire de l'éducation, méthodes actives, nature

Keywords : pedagogical innovation, primary education, history of education, active method, nature

\section{AUTEUR}

PIERRE DASI

Pierre Dasi est professeur agrégé en lycée. Il possède un doctorat en sciences de l'éducation (Laboratoire CIRNEF, Centre interdisciplinaire de Recherche Normand en Éducation et Formation - Université de Normandie). Sa thèse soutenue en 2018 à l'Université de Caen s'intitule : «Penser et représenter la nature à l'école primaire française des années 1870 aux années 1920 ». 